\title{
INTERFERÊNCIAS DE ESPÉCIES ARBÓREAS NA INTERCEPTAÇÃO DAS ÁGUAS PLUVIAIS URBANAS
} INTERFERENCES OF ARBOREAL SPECIES IN THE INTERCEPTION OF URBAN STORMWATER

\section{Patrícia Layne Alves}

Professora, Instituto Federal de Educação, Ciência e Tecnologia de Goiás (IFG/Goiânia). Coordenadora do Núcleo de Estudos e Pesquisas em Engenharia Civil e Meio Ambiente, IFG/Goiânia-Goiânia (GO), Brasil.

\section{Klebber Teodomiro Martins Formiga}

Professor assistente na Escola de Engenharia Civil, Universidade Federal de Goiás (UFG). Programa de Pósgraduação em Ciências Ambientais, UFG-Goiânia (GO), Brasil.

\section{Marco Antônio Borges Traldi}

Analista de Controle Externo do Tribunal de Contas do Estado de Goiás. Pesquisador colaborador do Núcleo de Estudos e Pesquisas em Engenharia Civil e Meio Ambiente, IFG/Goiânia-Goiânia (GO), Brasil.

\section{Endereço para correspondência:}

Patrícia Layne Alves - Instituto Federal de Educação, Ciência e Tecnologia de Goiás - Núcleo de Estudos e Pesquisas em Engenharia Civil e Meio Ambiente - Rua 75, 46 Centro - CEP 74055-110 Goiânia (GO), Brasil E-mail: patricia.alves@ifg.edu.br

Recebido: 15/12/2016

Aceito: 05/02/2018

\section{RESUMO}

Este estudo avaliou a interceptação e seus impactos nas bacias urbanas. A coleta de dados ocorreu em Uruaçu, Goiás, entre 2013 e 2014, e utilizou três indivíduos arbóreos. Para eventos com precipitações medianas de $20,7 \mathrm{~mm}$, nas três espécies encontraram-se os seguintes valores medianos para interceptação e escoamento pelo tronco, respectivamente: 5,7 e 0,1 mm para a Mangifera indica; 4,5 e 0,2 $\mathrm{mm}$ para a Licania tomentosa; e 3,8 e $0,3 \mathrm{~mm}$ para a Tabebuia ochracea. 0 tempo de retardo médio ocasionado pelas três espécies foi de três minutos. $O$ estudo comprovou a interferência e os impactos positivos das árvores na interceptação das águas pluviais em ambiente urbano, indicou a possibilidade de redução do escoamento em até $27 \%$ e mostrou que a arborização urbana pode reduzir o escoamento de águas pluviais e a intensidade do volume escoado, bem como aumentar o tempo de concentração e o tempo ao pico dos hidrogramas.

Palavras-chave: arborização urbana; precipitação interna; escoamento pelo tronco; hidrologia urbana.

\section{ABSTRACT}

This study evaluates evaluated the interception and its impacts in urban watersheds. Data collection occurred in Uruaçu, Goiás, between 2013 and 2014, and used three individual trees. For events with median rainfall of 20.7 $\mathrm{mm}$, the three species met the following median values for interception and stem flow, respectively: 5.7 and $0.1 \mathrm{~mm}$ for Mangifera indica; 4.5 and $0.2 \mathrm{~mm}$ for Licania tomentosa; and 3.8 and $0.3 \mathrm{~mm}$ for Tabebuia ochracea. The delay time of the surface runoff was on average $3 \mathrm{~min}$ for the three species. The study proves proved the interference and the positive impacts of treetops and trunks on the interception of rainwater in an urban environment. It indicates the possibility of annual runoff reduction in up to $27 \%$ and shows that urban afforestation can reduce stormwaterstorm water runoff and intensity of volume disposed, as well as increase the time of concentration and the time to the peak of hydrographs, especially of small watersheds.

Keywords: urban forestry; stem flow; through fall; urban hydrology. 


\section{INTRODUÇÃO}

O crescimento populacional e econômico das cidades transforma áreas naturais em paisagens urbanas e, com isso, surge uma série de problemas relacionados à gestão dos recursos hídricos. À medida que há uma urbanização desordenada das bacias hidrográficas, diversos desequilíbrios são ocasionados ao meio ambiente, resultando também em danos ao homem. A alteração de alguns processos inerentes ao ciclo hidrológico nesses ambientes é uma das consequências provocadas pela falta ou inobservância do planejamento da ocupação e do uso do solo.

O direcionamento de maior parcela de água pluvial para o escoamento superficial é um dos problemas ocasionados pela urbanização em decorrência da supressão da cobertura vegetal e impermeabilização do solo. $O$ aumento do volume escoado, bem como da vazão de pico, ocasiona a redução do tempo de concentração das bacias, provocando eventos de cheias cada vez mais críticos. Com isso, conforme apontam Fletcher et al. (2013) em suas pesquisas acerca da compreensão, gestão e modelagem da hidrologia urbana, há uma tendência entre os pesquisadores do tema em almejar a restauração do balanço hidrológico ao mais próximo possível dos cenários de pré-desenvolvimento, a fim de melhorar a capacidade de vida da paisagem.

A busca por ampliações nos tempos de concentração é algo valioso para amenizar os impactos e custos das obras de sistemas de drenagem (ALMEIDA et al., 2016). Com isso, a arborização urbana constitui-se um fator importante a ser considerado em modelagens ambientais, especialmente em pequenas bacias cujas interferências podem implicar ampliação do tempo ao pico do hidrograma, aumento no tempo de retardo e redução na intensidade do escoamento superficial (ALVES, 2015).

As árvores, inseridas na paisagem urbana, fornecem muitos benefícios sociais, psicológicos e econômicos por meio da promoção do bem-estar, da redução da temperatura, da poluição, e das transformações nos processos hidrológicos (GÓMEZ-BAGGETHUN \& BARTON, 2013). Berland e Hopton (2014) e Inkiläinen et al. (2013) apontam que a arborização desempenha um importante papel nos sistemas de drenagem das águas pluviais urbanas por meio da interceptação pelas copas. $O$ volume interceptado reduz a intensidade do pico dos fluxos de escoamento. Ademais, conforme
Levia e Germer (2015), nesse processo, a arquitetura das árvores e as propriedades da casca influenciam grandemente a proporção de precipitação que pode ser interceptada e escoada pelo tronco.

Sabe-se que a interceptação da água da chuva ocorre tanto em ambientes florestais quanto em indivíduos isolados, quando esta é subdividida nas copas das árvores, onde, temporariamente, uma parte é retida, outra escoa pelo tronco e outra é evaporada para atmosfera (FREITAS et al., 2016; XIAO \& MCPHERSON, 2016). Segundo Xiao e McPherson (2016) e Li et al. (2013), o processo de interceptação nas copas é influenciado por três fatores principais: o tipo de evento de chuva (magnitude, intensidade e duração); a estrutura da copa em cada espécie arbórea; e as condições meteorológicas.

A maioria dos estudos em sistemas florestais naturais ou gerenciados indica que a intercepção pode representar 10-50\% da precipitação (VAN DIJK et al., 2015). No entanto, há informações conflitantes sobre o processo pelo qual a intercepção interage com suas variáveis. Por exemplo: não há consenso quanto ao efeito da intensidade de precipitação na intercepção. Alguns estudos sugerem que ela aumenta com a intensidade do evento em razão da saturação gradual da copa (LIVESLEY et al., 2014), enquanto outros mostram que as intensidades mais elevadas resultam em menor intercepção em decorrência da "agitação" das copas (INKILÄINEN et al., 2013).

Além disso, os estudos de intercepção geralmente envolvem grandes faixas florestais ou árvores adultas individuais, condições em que muitas características das estruturas arbóreas não podem ser aferidas precisamente (por exemplo, área foliar, densidade de ramos etc.) (LEVIA et al., 2015). Como resultado, a relação entre intercepção e características arbóreas ainda permanece obscura (NANKO et al., 2013). Parte desse problema se deve ao fato de que aferir a interceptação em ambientes urbanos é um objetivo complexo de ser alcançado, e muitos estudos, como os de Xiao et al. (2000), Xiao e Mcpherson (2002), Silva et al. (2010) e Livesley et al. (2014), utilizaram pluviômetros ou recipientes graduados para quantificá-la. Procedendo dessa forma ficam impossibilitadas as análises da duração, do tempo de ocorrência e da intensidade dos eventos chuvosos. 
A necessidade de caracterizar as chuvas quanto a distribuição temporal, volume precipitado e variações de intensidades é de suma importância porque as interceptações ocasionadas pelas copas das árvores não acontecem de maneira constante ao longo do evento chuvoso. Holder e Gibbes (2017) destacam que a interceptação também é variável de evento para evento. Além dessas variações em relação às chuvas, nos sistemas urbanos, a cobertura pelas copas é descontínua, as árvores são frequentemente isoladas e há alto número de variedade de espécies, tipo de copa/folha e de suas características, ampliando a dificuldade e a complexidade de se determinar parâmetros exatos para o tema (Ll et al., 2016).

As dificuldades inerentes ao assunto levam a uma grande variação de metodologias e critérios analisados. Grande parte das pesquisas são realizadas em ambientes internacionais, a exemplo de Xiao et al. (2000), que desenvolveram em Davis, Califórnia (EUA), um modelo tridimensional e estimaram, em eventos de precipitação de $8,8 \mathrm{~mm}$, a interceptação em 1,1 $\mathrm{mm}$ e 2,9 $\mathrm{mm}$ para Pyrus calleryana e Quercus suber, respectivamente, e o escoamento pelo tronco em torno de 0,5 $\mathrm{mm}$ e $1,1 \mathrm{~mm}$. Na mesma localidade, Xiao e McPherson (2016) aferiram a capacidade de interceptação de 20 espécies de árvores urbanas em um simulador de precipitação. As intensidades simuladas variaram de 3,5 a $139,5 \mathrm{~mm} \cdot \mathrm{h}^{-1}$. As características arbóreas foram obtidas por meio do método de análise de imagem, e os resultados indicaram que as interceptações médias em todas as espécies foram $0,86 \mathrm{~mm}$. As abordagens de ambos os estudos permitiram estimativas de boa qualidade, porém só podem ser aplicadas a situações de pesquisa controlada e para copas sem a interferência de vegetações adjacentes ou estruturas construídas. Um método mais simples para a determinação da interceptação da copa/dossel e do escoamento pelo tronco foi desenvolvido por Livesley et al. (2014) para a medição em ambientes urbanos reais com árvores adultas na cidade de Melbourne, Victoria (Austrália). Desta pesquisa obteve-se, em eventos de precipitação média de $12 \mathrm{~mm}$, para duas espécies de eucalipto, Eucalyptus nicholii e Eucalyptus saligna, uma interceptação de 3,88 e 3,03 $\mathrm{mm}$ respectivamente; quanto ao escoamento pelo tronco, foi obtido um desempenho ínfimo para a Eucalyptus nicholii e a Eucalyptus saligna, a qual, por possuir casca mais lisa, só registrou cerca de $2 \mathrm{~mm}$.

No Brasil, Silva et al. (2010), em estudos realizados no campus da Escola Superior de Agricultura "Luiz de Queiroz" da Universidade de São Paulo (ESALQ/USP), São Paulo, envolvendo duas espécies, a Caesalpinia pluviosa e a Tipuana tipu, concluíram que ambas apresentam grande potencial para interceptação, principalmente em eventos mais duradouros e com precipitações superiores a $20 \mathrm{~mm}$, tendo a Caesalpinia pluviosa ou sibipiruna obtido em média $4,5 \mathrm{~mm}$ de interceptação e a Tipuana tipu ou tipuana, média de 10,3 mm.

As diferentes características das copas (densa ou esparsa), das folhas (simples ou compostas, persistente ou caduca) e do tronco (liso ou áspero) influenciam na interceptação, na redistribuição e no escoamento das chuvas, e constituem parâmetros diferenciais para a simulação dos processos hidrológicos em uma bacia urbana.

No entanto, ainda são poucos os estudos que relacionam as interceptações das chuvas pelas árvores aos modelos hidrológicos de bacias. Hilde e Paterson (2014) salientam que os modelos de interceptações e a previsão de seus efeitos sobre o clima e os recursos hídricos devem ser inseridos nas modelagens ambientais. Porém, para isso, exige-se uma compreensão baseada no impacto das árvores e em suas particularidades sobre o processo de drenagem para a inserção das características de interceptação pelas copas das árvores em simulações computacionais que utilizam modelos como UFORE-Hydro (YANG et al., 2011), i-Tree (HILDE \& PATERSON, 2014), SWMM (KREBS et al., 2013) e TOPLATS (BORMANN, 2006).

Inkiläinen et al. (2013) e Alves (2015) afirmam que as árvores podem ser uma alternativa para a redução do escoamento urbano, pois sua presença reduz significativamente o escoamento, principalmente em eventos de baixa intensidade e curta duração, e podem ampliar o tempo de concentração das bacias. Com o intuito de avaliar e quantificar a interceptação das águas pluviais ocasionada pela presença das árvores no meio urbano, no tempo ao pico e na intensidade do volume escoado em bacias urbanas, realizou-se uma pesquisa na cidade de Uruaçu, Goiás. Para a coleta de dados, foram utilizados pluviógrafos e coletores no tronco em três espécies arbóreas diferentes. 


\section{MATERIAIS E MÉTODOS}

O estudo foi realizado em uma área urbana da cidade de Uruaçu, Goiás (1431'6.23"S, 498'11.16"O), região climática que, de acordo com a classificação de Köppen, é do tipo Aw com clima tropical, estação seca prolongada e chuvas concentradas no verão. Segundo o banco de dados Hidroweb da Agência Nacional de Águas (ANA, 2014), a precipitação anual no município é de aproximadamente $1.006,8 \mathrm{~mm}$ e, conforme o Instituto Nacional de Meteorologia (INMET, 2014), a temperatura média dos meses mais quentes é de 39,4 으, enquanto a dos meses mais frios é de 10,8 으. Em 2013/2014, durante os cinco meses de medição, em relação aos dados pluviométricos, o mínimo mensal foi de $17,5 \mathrm{~mm}$ (fevereiro de 2014) e o máximo mensal de 182,4 mm (dezembro de 2013).

Três indivíduos arbóreos, sendo um em grupo (Mangifera indica) e dois isolados (uma Tabebuia ochracea e uma Licania tomentosa), foram selecionados em razão dos maiores diâmetros de altura do peito (DAP) entre os outros exemplares de suas espécies existentes no local da pesquisa, por serem amplamente empregados na arborização urbana regional e por suas características de copa, casca e folhagem.

Tabela 1 - Parâmetros arbóreos dos indivíduos estudados na pesquisa.

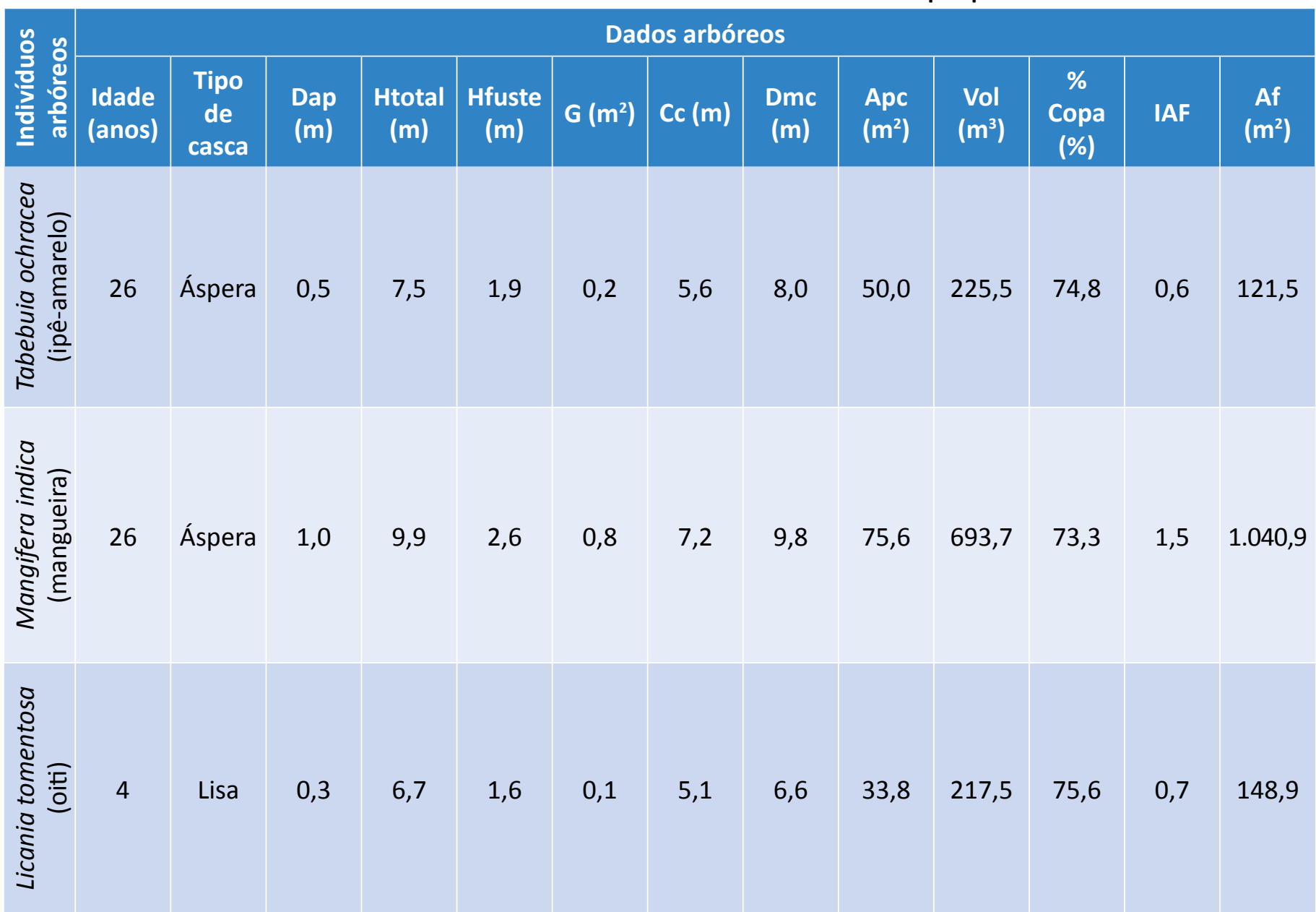

Idade (anos): idade de plantio do indivíduo; Dap (cm): diâmetro a altura do peito; Htotal $(\mathrm{m})$ : altura total da árvore; Hfuste (m): altura de fuste (tronco); $\mathrm{G}\left(\mathrm{m}^{2}\right)$ : área basal da árvore $=\left(\pi^{*}\right.$ dap $\left.{ }^{2}\right) / 4 ; \mathrm{Cc}(\mathrm{m})$ : comprimento de copa; Dmc $(\mathrm{m})$ : diâmetro médio de copa; $\mathrm{Apc}\left(\mathrm{m}^{2}\right)$ : área de projeção de copa $=(\pi / 4) * \mathrm{dmc}^{2} ; \mathrm{Vol}\left(\mathrm{m}^{3}\right)$ : volume de copa; \%copa: percentagem de copa $=(\mathrm{cc} /$ htotal $) * 100 ; \mathrm{IAF}$ : Índice de área foliar; Af $\left(\mathrm{m}^{2}\right)$ : área foliar. 
Os parâmetros arbóreos das espécies estão expressos na Tabela 1 e foram necessários na análise das características dos indivíduos arbóreos e de seu desempenho quanto à interceptação.

A precipitação bruta (PB) e a precipitação interna (PI) foram aferidas por quatro pluviógrafos do modelo RG3-M, marca Onset, instalados a 1,50 m de altura do solo, de modo que três ficaram posicionados sob as copas das árvores e um em campo aberto. Os equipamentos foram instalados durante o período de novembro de 2013 a março de 2014 e tiveram suas localizações sob as copas alteradas a cada 15 dias a fim de obter dados mais representativos. Os registros dos pluviógrafos foram adquiridos com uma discretização do intervalo de tempo em 1 minuto.

O escoamento pelo tronco (ET) foi obtido por meio da construção de estruturas nas árvores que desaguavam em galões com capacidade de $23 \mathrm{~L}$ (Figura 1). Tais estruturas para captação das águas foram executadas com calhas de borracha seladas ao tronco por meio da aplicação de espuma de poliuretano.

Seguindo a metodologia proposta por Livesley et al. (2014), a interceptação pelas copas (Cl) para cada evento chuvoso foi calculada conforme a Equação 1:
$C I=P B-(P I+E T)$

Em que:

$\mathrm{Cl}=$ interceptação pelas copas;

$\mathrm{PB}=$ precipitação bruta (precipitação acumulada total em cada evento chuvoso medido no pluviógrafo em campo aberto) (mm);

$\mathrm{PI}=$ precipitação total acumulada sob a copa; e $\mathrm{ET}=$ escoamento total pelo tronco.

A medição temporal dos eventos chuvosos com o uso dos pluviógrafos permitiu a padronização dos dados considerando que as chuvas ocorridas sob a copa da árvore tivessem a mesma duração e o mesmo momento de início e final que as chuvas ocorridas em campo aberto.

Durante o período experimental, foram registrados 104 eventos com variações de chuvas com intensidade média de $1,2 \mathrm{~mm} \cdot \mathrm{h}^{-1}$ até $38 \mathrm{~mm} \cdot \mathrm{h}^{-1}$, e precipitação acumulada total variando entre 0,6 e $68,4 \mathrm{~mm}$. Para o presente estudo, foram selecionados os 42 eventos chuvosos que tiveram precipitações bruta acima de $5 \mathrm{~mm}$.
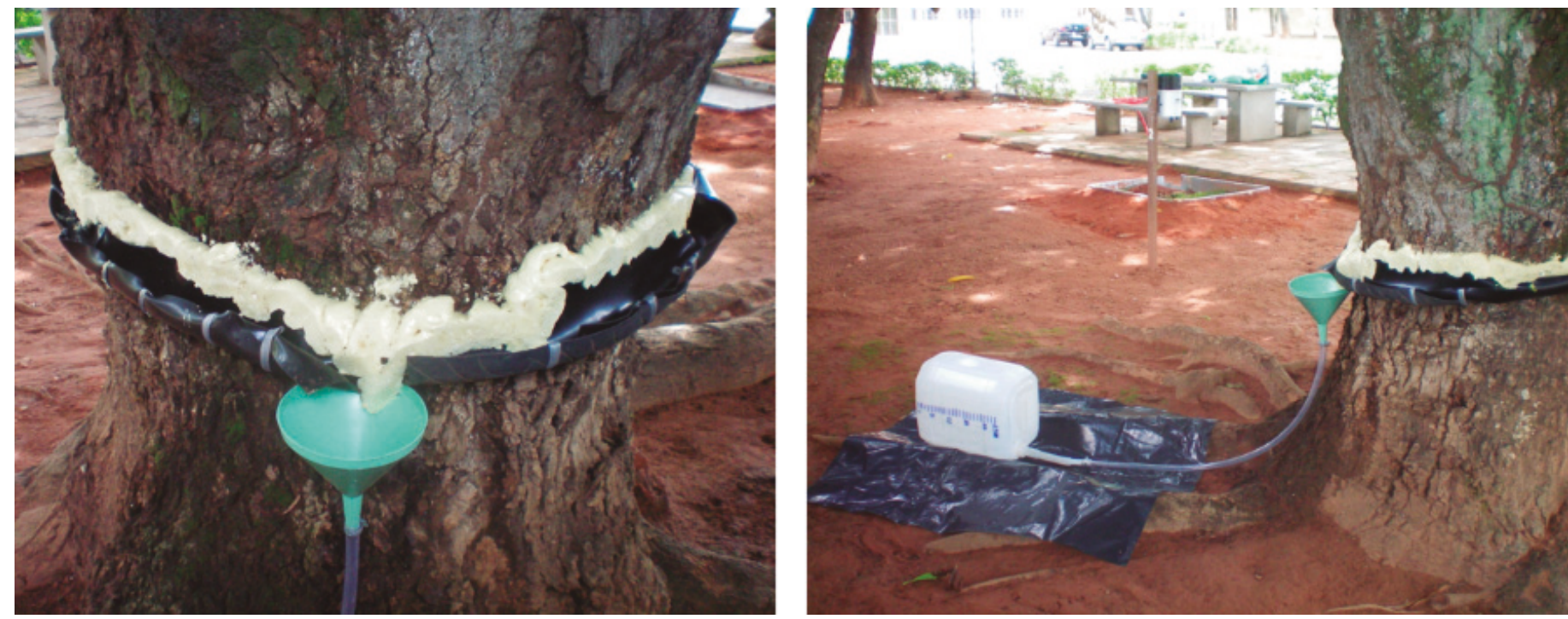

Figura 1 - Imagem de uma das estruturas para captação do escoamento pelo tronco. 


\section{RESULTADOS E DISCUSSÃO}

De modo geral, nota-se que há um comportamento variável entre $\mathrm{PI}+\mathrm{ET}$ e $\mathrm{Cl}$ nos eventos chuvosos considerados nas análises. Quanto à relação entre os eventos chuvosos e as $\mathrm{Cl}$ (Figura 2), assim como em PI + ET, as medianas variaram de forma aleatória; de modo geral, mantiveram-se em grande parte com valores abaixo de $10 \mathrm{~mm}$ e apenas alguns eventos chuvosos com comportamento diferente. Destaca-se o evento 94, que obteve a maior interceptação mediana, $13,3 \mathrm{~mm}$, intensidade média de $31,6 \mathrm{~mm} \cdot \mathrm{h}^{-1}$, precipitação acumulada de $29 \mathrm{~mm}$, duração de 55 minutos e precipitação mediana acumulada sob as copas de $18,3 \mathrm{~mm}$. Ao contrário, o evento 73 foi o de menor interceptação mediana de $\mathrm{Cl}, 0,64 \mathrm{~mm}$, intensidade média de $11,6 \mathrm{~mm} \cdot \mathrm{h}^{-1}$, precipitação acumulada total de $6,2 \mathrm{~mm}$, duração de 32 minutos e precipitação mediana acumulada sob as copas de $5,5 \mathrm{~mm}$.

Na Figura 3 fica evidente que poucos eventos chuvosos tiveram seus valores medianos de PI + ET superiores a $10 \mathrm{~mm}$. Destaca-se apenas o evento 100 que obteve valor mediano de PI + ET de $57 \mathrm{~mm}$. A intensidade média desse evento foi de $27,2 \mathrm{~mm} \cdot \mathrm{h}^{-1}$, precipi- tação acumulada de 68,4 mm, duração de 151 minutos e precipitação mediana acumulada sob as copas de $56,2 \mathrm{~mm}$.

Foi identificada pouca correlação linear $\left(R^{2}=0,30\right)$ entre a $\mathrm{PB}$ e a $\mathrm{Cl}$ nas três árvores envolvidas na pesquisa. Não há uma relação direta entre $\mathrm{PB}$ e $\mathrm{Cl}$ nos eventos chuvosos considerados nas análises, comportamento que pode ser explicado pelas divergências entre as características arbóreas das espécies e dos eventos chuvosos.

A capacidade de interceptação de chuvas em indivíduos arbóreos é influenciada por diversos aspectos, entre os quais podem-se destacar:

- intensidade e duração da chuva;

- espécie arbórea;

- características arbóreas da espécie;

- temperatura e umidade relativa do ar;

- poda/manutenção dos indivíduos.
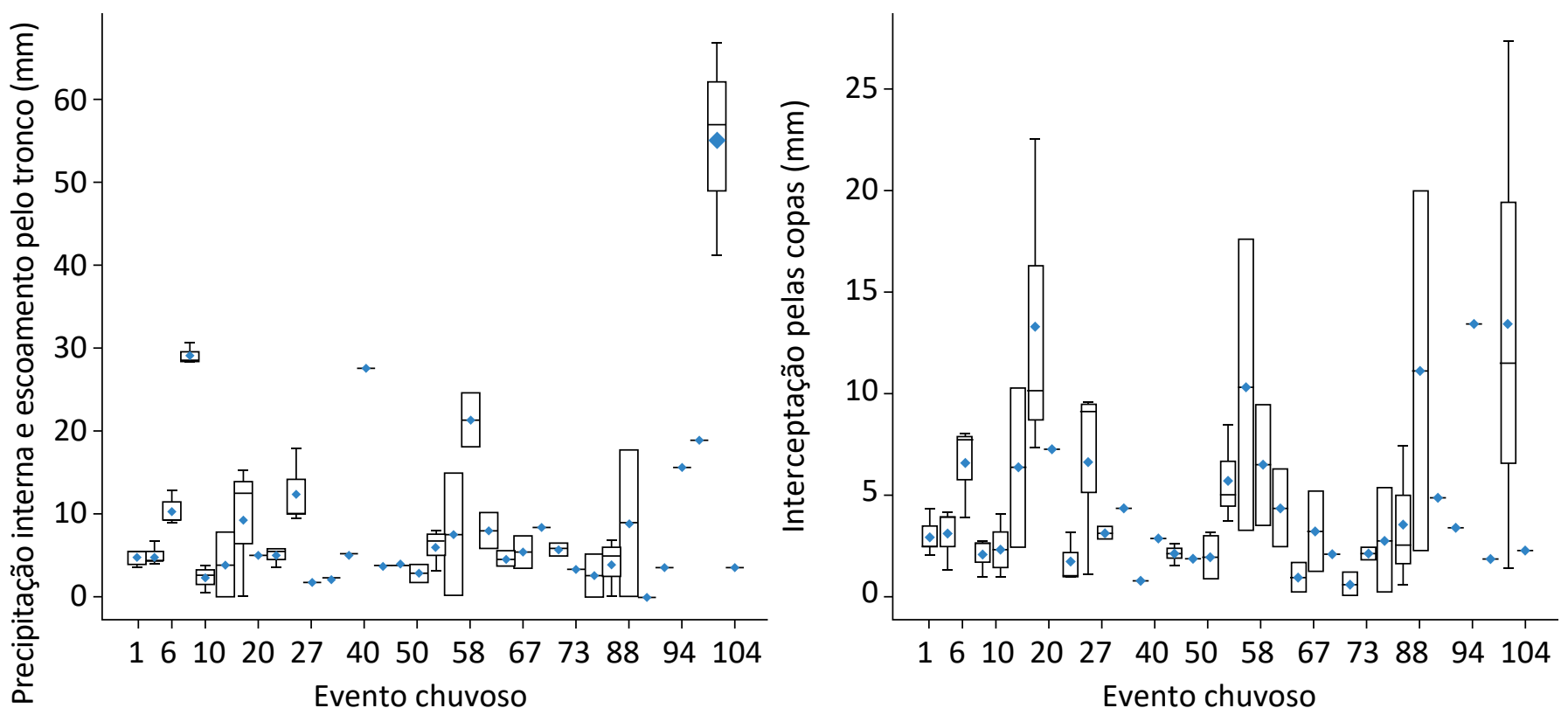

PI + ET: precipitação interna e escoamento pelo tronco $(\mathrm{mm})$; $\mathrm{Cl}$ : interceptação pelas copas $(\mathrm{mm})$.

Figura 2 - Box plot das precipitações internas + escoamento pelo tronco e interceptações pelas copas nos três indivíduos arbóreos envolvidos na pesquisa durante os $\mathbf{4 2}$ eventos chuvosos considerados nas análises. 
Nos eventos chuvosos analisados quanto à $\mathrm{Cl}, \mathrm{PB}$ e $\mathrm{PI}$ (Figura 3), o desempenho das copas das árvores para interceptação $(\mathrm{Cl})$ obtiveram valores medianos de 3,8, 4,5 e 5,7 mm para a Tabebuia ochracea (ipê amarelo), a Licania tomentosa (oiti) e a Mangifera indica (mangueira) respectivamente. A PB aferida pelo pluviógrafo externo teve valor mediano de $20,7 \mathrm{~mm}$, enquanto as PI das mesmas espécies foram de 13,0, 10,2 e 13,3 mm.
O ET (Figura 3) foi na Tabebuia ochracea (ipê amarelo) o menor valor mediano, 0,1 mm; na Mangifera indica (mangueira), o valor de 0,2 $\mathrm{mm}$; e na Licania tomentosa (oiti) o maior valor maior obtido, 0,3 $\mathrm{mm}$.

Quanto ao retardo no tempo de início do evento chuvoso (R) (Figura 3), este foi de 2 minutos para Mangifera indica (mangueira) e Tabebuia ochracea (ipê amarelo). e de 3 minutos para a Licania tomentosa (oiti).
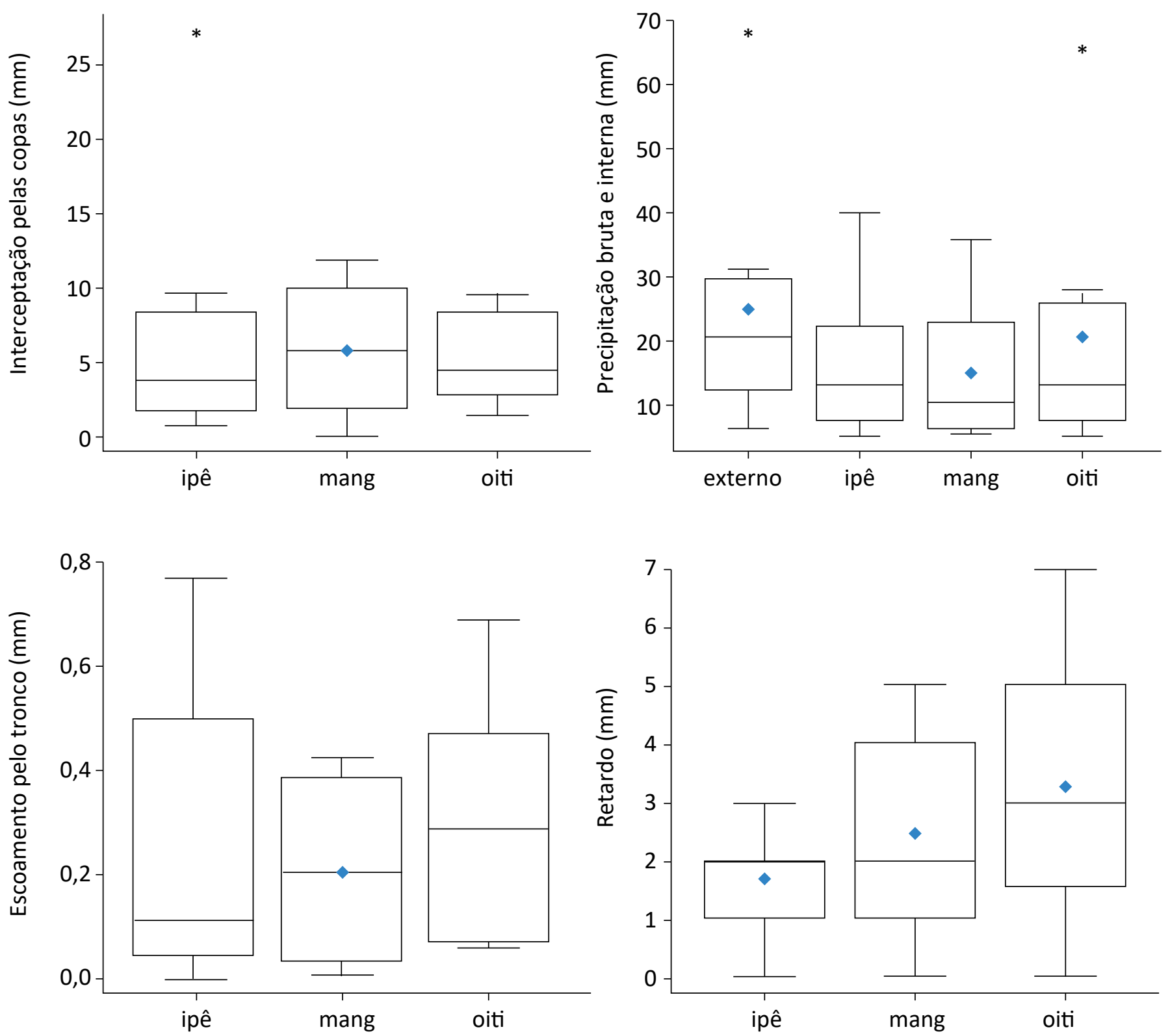

$\mathrm{Cl}$ : interceptação pelas copas (mm); PB: precipitação bruta $(\mathrm{mm})$; PI: precipitação interna $(\mathrm{mm})$; ET: escoamento pelo tronco $(\mathrm{mm})$; R: retardo no tempo de início do evento chuvoso ( $\mathrm{min}$ ).

Figura 3 - Box plot dos 42 eventos chuvosos analisados em relação à CI, PB, PI, ET e R. 
A Mangifera indica (mangueira) foi o indivíduo arbóreo que apresentou melhor desempenho quanto ao $\mathrm{Cl}$ e isso é explicado pelo fato de o exemplar possuir o maior volume de copa e índice e área foliar entre as espécies estudadas (Tabela 1), estar em grupo e se caracterizar por folhas simples, permanente e glabras e casca áspera. A Licania tomentosa (oiti) tem características folhosas similares à mangueira, porém com casca lisa. Tal característica arbórea explica seu melhor desempenho quanto ao escoamento pelo tronco, do mesmo modo que seu melhor desempenho em relação ao retardo pode ser explicado por sua copa densa com folhas e galhos intensamente entrelaçados favorecendo a retenção por maior tempo das águas de chuva por suas copas. A Tabebuia ochracea (ipê amarelo), por sua vez, obteve o menor desempenho quanto a $\mathrm{Cl}, \mathrm{Pl}$ e ET, o que é justificado pelo fato de a espécie apresentar folhas compostas e pilosas e casca áspera, aspectos que reunidos favorecem o armazenamento de água na copa $(\mathrm{Cl})$ e reduzem a $\mathrm{PI}$ e ET.
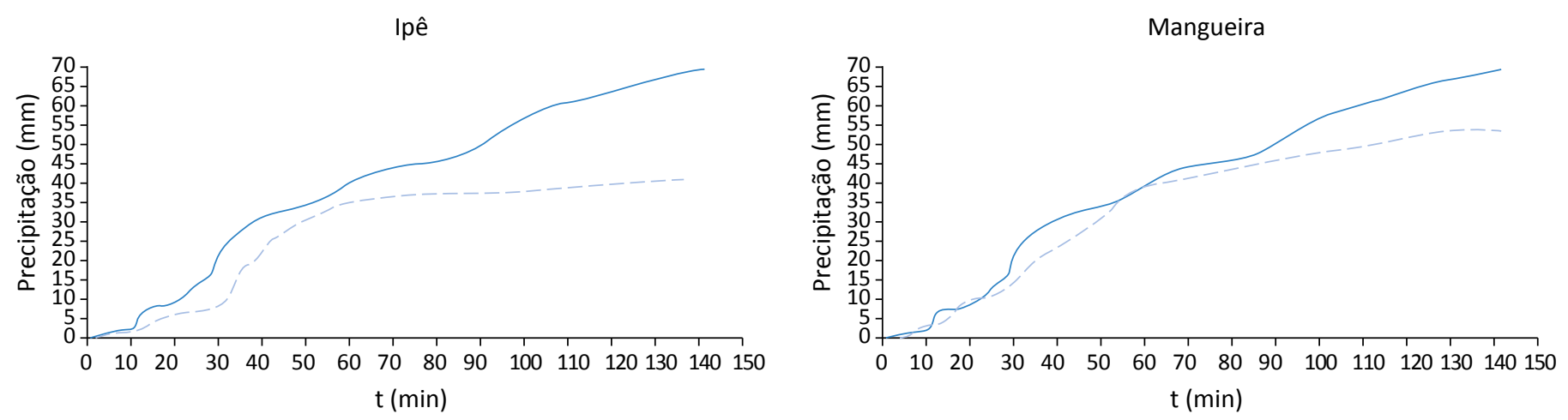

Oiti

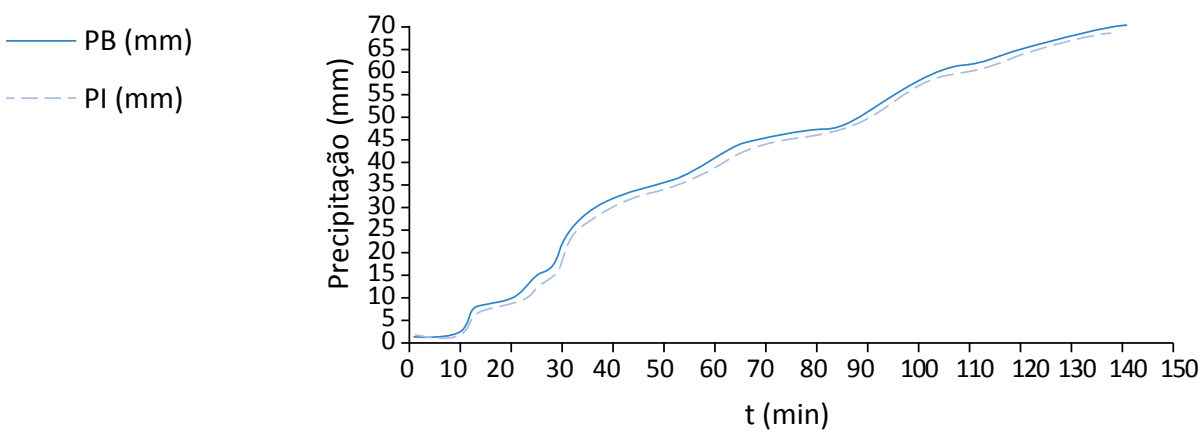

PB: Precipitação bruta; PI: Precipitação interna.

Figura 4 - Hietogramas do evento chuvoso ocorrido nos dias 3 de março de 2014 a 4 de março de 2014, de $22 \mathrm{~h} 50$ à $1 \mathrm{~h} 20$, demonstrando o comportamento quanto à precipitação interna (mm) e precipitação bruta (mm). 
A Figura 5 expõe o comportamento do tempo ao pico do evento de maior PB $(68,4 \mathrm{~mm})$. Nos hietogramas é notória a diferença entre a $\mathrm{PB}$ registrada pelo pluviógrafo externo e as $\mathrm{PI}$ aferidas sob as copas. O pico do evento (PB) ocorreu em campo aberto aos $30 \mathrm{mi}-$ nutos, com precipitação de $3,8 \mathrm{~mm}$. Nos exemplares arbóreos houve uma redistribuição desse valor máximo; por exemplo, no Ipê amarelo houve três outros sucessivos picos menores, com valores inferiores a $2 \mathrm{~mm}$ ocorridos a 35, 40 e 46 minutos respectivamente. Comportamento semelhante pode ser observado tanto na mangueira quanto no oiti. Logo, fica evidente a ampliação do tempo ao pico, bem como a redução de sua intensidade em todas as espécies. A amplitude dos valores identificados na pesquisa poderá ser expandida à medida que novos exemplares forem associados em uma mesma área urbana, permitindo assim amortização de cheias e redução de diâmetros de tubulação dos sistemas de drenagem urbana.
Obter uma metodologia-padrão para medir a interceptação pelas copas é extremamente difícil tendo em vista que os estudos são realizados em regiões de climas diversos utilizando espécies arbóreas diferentes (SOTO-SCHÖNHERR \& IROUMÉ, 2016; Ll et al., 2016).

A precipitação mediana dos eventos analisados foi de $20,7 \mathrm{~mm}$. De modo geral, as interceptações medianas obtidas nos indivíduos arbóreos envolvidos na pesquisa foram de 4,5 mm. A Mangifera indica (mangueira) teve melhor interceptação em razão de suas características de arquitetura da copa, da casca e das folhas. Quanto ao retardo no tempo de início do evento chuvoso e o volume escoado pelo tronco, a Licania tomentosa (oiti) obteve os maiores valores em decorrência do fato de ser a única espécie aqui estudada com casca lisa. Em geral, a interceptação das espécies foi diretamente relacionada às suas características arbóreas, em especial a arquitetura da copa e particularidades das folhas e da casca.
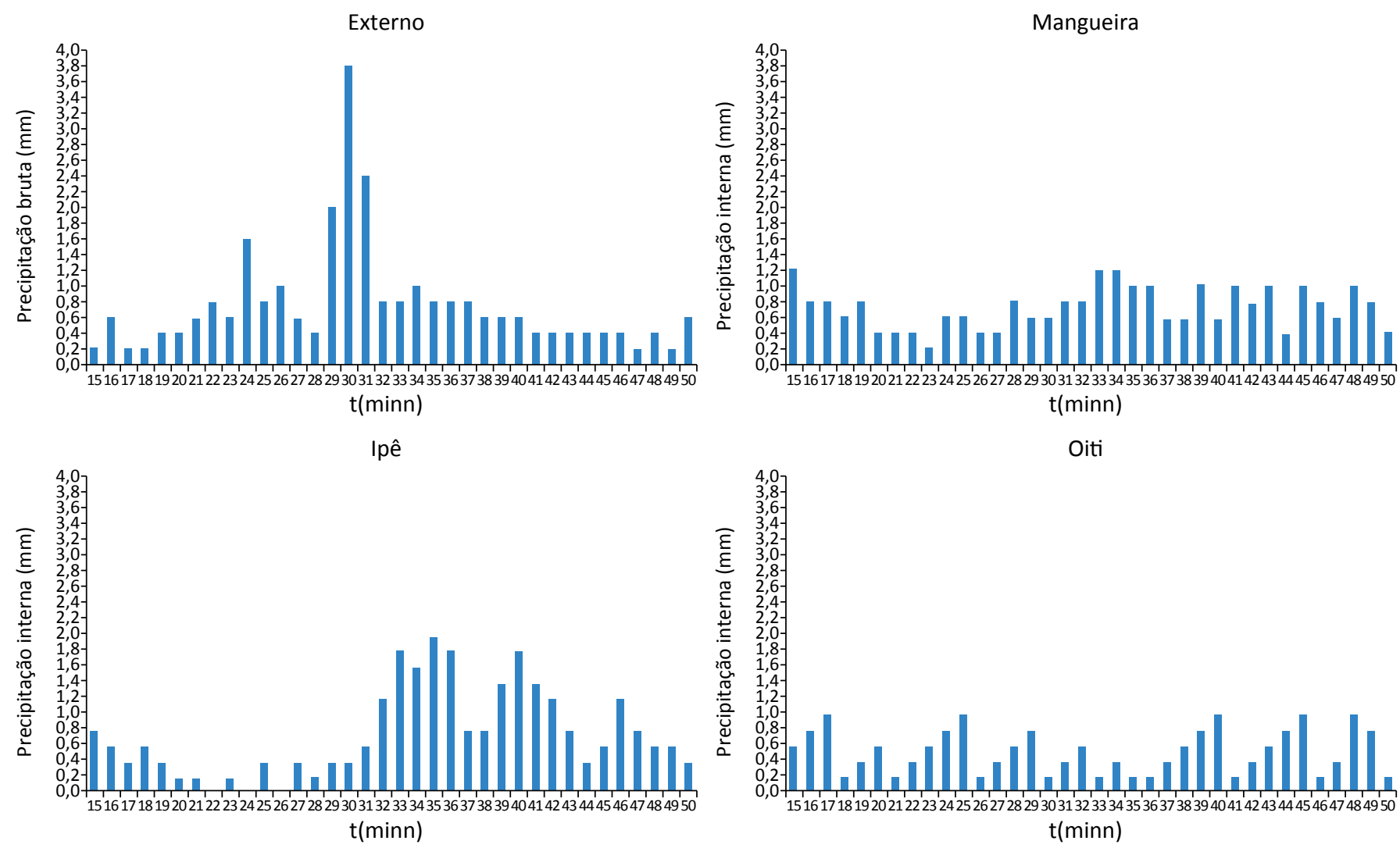

Figura 5 - Hietogramas de trecho do evento chuvoso ocorrido nos dias 3 de março de 2014 a 4 de março de 2014 , de $22 \mathrm{~h} 50$ à $1 \mathrm{~h} 20$, demonstrando o comportamento variável de tempo ao pico nas espécies arbóreas. 
A presença dessas árvores tem um grande significado em áreas urbanas, pois para o caso estudado houve uma redução de até $27 \%$ do volume precipitado durante chuvas ocorridas no município de Uruaçu, Goiás. Tal volume, sem a presença de árvores, escoaria diretamente para os sistemas de drenagem.

A capacidade de interceptação por parte da arborização urbana é ressaltada com os resultados aqui obtidos por serem próximos aos encontrados por Xiao et al. (2000), que constataram, em eventos de precipitação de $8,8 \mathrm{~mm}$, a interceptação média de $2 \mathrm{~mm}$ e o escoamento pelo tronco em torno de $0,8 \mathrm{~mm}$. Silva et al. (2010) também concluíram que em eventos mais duradouros e com precipitações superiores a $20 \mathrm{~mm}$ as interceptações são em média 7,4 mm.

Quanto aos efeitos das intensidades nas interceptações, em geral, na presente pesquisa, em eventos de baixa intensidade, de $1,2 \mathrm{~mm} \cdot \mathrm{h}^{-1}$ até $38 \mathrm{~mm} \cdot \mathrm{h}^{-1}$, a interceptação foi superior, em média $4,5 \mathrm{~mm}$. Já os resultados obtidos por Xiao \& McPherson (2016) comprovaram que em intensidades maiores, de 3,5 a 139,5 mm.h ${ }^{-1}$, as interceptações eram inferiores, em média 0,86 mm.

O estudo evidencia que a arborização urbana faz com que copas, ramos, troncos e raízes funcionem como obstáculos naturais para as águas das chuvas que, com uma retenção temporária, alcançam mais lentamente os corpos receptores, promovendo a ampliação do tempo de concentração, do tempo de retardo e consequentemente do tempo ao pico de vazão.

Uma das consequências da urbanização é o aumento do volume escoado e das vazões de pico, ao mesmo tempo em que ocorre a redução do tempo de concentração, provocando eventos de cheias cada vez mais críticos (DU et al., 2012). As espécies arbóreas aqui estudadas apresentaram um tempo de retardo médio de 3 minutos, podendo chegar a até 15 minutos em eventos com menor intensidade. Ocasionaram também uma redistribuição dos picos de vazão, ampliando o tempo ao pico, bem como a redução de sua intensidade. Esses dados demonstram que as árvores colaboram para o aumento no tempo de concentração nas bacias hidrográficas urbanas, podendo assim minimizar as cheias, inundações e seus prejuízos.

Os valores aparentemente pequenos relativos ao tempo de retardo são significativos quando associados às pequenas bacias hidrográficas, pois, em bacias cujo tempo de concentração é, por exemplo, em torno de 5 minutos, uma redução de 2 ou 3 minutos teria impactos relevantes nos sistemas de drenagem e corpos receptores.

\section{CONCLUSÕES}

A presente pesquisa explorou a relação entre a capacidade de interceptação por parte de três espécies arbóreas e as mudanças que estas podem causar no funcionamento hidrológico urbano, obtendo quatro resultados principais:

1. a capacidade de interceptação variou entre as espécies, confirmando a importância de aplicar esse tipo de pesquisa para outras localidades e espécies de árvores urbanas;

2. por causa das diferenças na arquitetura arbórea, folha (índice e área foliar) e casca, espécies com copas mais frondosas, densas e cascas ásperas tiveram maiores capacidades de interceptação;

3. as interceptações são superiores em eventos de baixa intensidade e curta duração;
4. há uma ampliação do tempo ao pico, bem como a redução de sua intensidade em todas as espécies, independentemente de suas características arbóreas.

Tais conclusões foram elucidadas por meio do monitoramento contínuo de interceptação/armazenamento das copas e do escoamento pelo tronco, e, com isso, este trabalho comprovou a importância da presença das árvores para os estudos hidrológicos urbanos. Para eventos com precipitação média de $20,7 \mathrm{~mm}$, a interceptação média ocasionada pelas espécies de Mangifera indica, Tabebuia ochracea e Licania tomentosa foi em média de $4,5 \mathrm{~mm}$, equivalendo a uma redução de até $27 \%$ das águas pluviais que atingiriam a superfície do solo.

Quantificar o potencial de diferentes espécies arbóreas para interceptar a precipitação sob uma variedade de 
condições meteorológicas é fundamental para validar a relação custo-eficácia do plantio de árvores como estratégia de infraestrutura verde. Ademais, as implicações à hidrologia urbana das interceptações pelas copas e pelo escoamento pelo tronco dependem do contexto da paisagem em que são inseridos. Em uma paisagem urbana com solo impermeável, com a cobertura arbórea de espécies semelhante às aqui estudadas, pode-se esperar uma redução significativa das águas pluviais que atingirão diretamente a superfície, bem como do escoamento, e o aumento dos tempos de concentrações das bacias.

\section{AGRADECIMENTOS}

Os autores expressam seus agradecimentos a: Fundação de Amparo à Pesquisa do Estado de Goiás (FAPEG) por conceder uma bolsa de estudo para o primeiro autor; Financiadora de Estudos e Projetos (FINEP) pelo auxílio financeiro para a compra de equipamentos; e Conselho Nacional de Desenvolvimento Científico e Tecnológico (CNPq) pela concessão de bolsa de Produtividade em Desenvolvimento Tecnológico e Extensão Inovadora - Nível 2 ao segundo autor.

\section{REFERÊNCIAS}

AGÊNCIA NACIONAL DE ÁGUAS (ANA). HidroWeb: sistemas de informações hidrológicas. Disponível em: <http:// hidroweb.ana.gov.br/HidroWeb>. Acesso em: 23 nov. 2014.

ALMEIDA, I. K. de; ALMEIDA, A. K.; STEFFEN, J. L.; ALVES SOBRINHO, T. Model for estimating the time of concentration in watersheds. Water Resources Management, v. 30, n. 12, p. 4083-4096, 2016. DOI: 10.1007/s11269-016-1383-x

ALVES, P. L. Capacidade de interceptação pelas árvores e suas influências no escoamento superficial urbano. Tese (Doutorado) - Universidade Federal de Goiás, Goiânia, 2015.

BERLAND, A.; HOPTON, M. E. Comparing street tree assemblages and associated stormwater benefits among communities in metropolitan Cincinnati, Ohio, USA. Urban Forestry \& Urban Greening, v. 13, n. 4, p. 734-741, 2014. DOI: 10.1016/j.ufug.2014.06.004

BORMANN, H. Impact of spatial data resolution on simulated catchment water balances and model performance of the multi-scale TOPLATS model. Hydrology and Earth System Sciences Discussions, v. 10, p. 165-179, 2006.

DU, J.; QIAN, L.; RUI, H.; ZUO, T.; ZHENG, D.; XU, Y.; XU, C. Y. Assessing the effects of urbanization on annual runoff and flood events using an integrated hydrological modeling system for Qinhuai River basin, China. Journal of Hydrology, $v$. 464-465, p. 127-139, 2012. DOI: 10.1016/j.jhydrol.2012.06.057

FLETCHER, T. D.; ANDRIEU, H.; HAMEL, P. Understanding, management and modelling of urban hydrology and its consequences for receiving waters: A state of the art. Advances in Water Resources, v. 51, p. 261-279, 2013. https:// doi.org/10.1016/j.advwatres.2012.09.001

FREITAS, J. P. O.; DIAS, H. C. T.; SILVA, E.; TONELLO, K. C. Net precipitation in a semideciduous forest fragment in Viçosa city, MG. Revista Árvore, Viçosa, v. 40, n. 5, p. 793-801, 2016. http://dx.doi.org/10.1590/0100-67622016000500003

GÓMEZ-BAGGETHUN, E.; BARTON, D. N. Classifying and valuing ecosystem services for urban planning. Ecological Economics, v. 86, p. 235-245, 2013. https://doi.org/10.1016/j.ecolecon.2012.08.019

HILDE, T.; PATERSON, R. Integrating ecosystem services analysis into scenario planning practice: Accounting for street tree benefits with i-Tree valuation in Central Texas. Journal of Environmental Management, v. 146, p. 524-534, 2014. https://doi.org/10.1016/j.jenvman.2014.05.039

HOLDER, C. D.; GIBBES, C. Influence of leaf and canopy characteristics on rainfall interception and urban hydrology. Hydrological Sciences Journal, v. 62, n. 2, p. 182-190, 2017. https://doi.org/10.1080/02626667.2016.1217414 
INKILÄINEN, E. N.; MCHALE, M. R.; BLANK, G. B.; JAMES, A. L.; NIKINMAA, E. The role of the residential urban forest in regulating throughfall: A case study in Raleigh, North Carolina, USA. Landscape and Urban Planning, v. 119, p. 91-103, 2013. https://doi.org/10.1016/j.landurbplan.2013.07.002

INSTITUTO NACIONAL DE METEOROLOGIA (INMET). BDMEP - Banco de Dados Meteorológicos para Ensino e Pesquisa. Disponível em: <http://www.inmet.gov.br/portal/index.php?r=bdmep/bdmep>. Acesso em: 23 nov. 2014.

KREBS, G.; KOKKONEN, T.; VALTANEN, M.; KOIVUSALO, H.; SETÄLÄ, H. A high resolution application of a stormwater management model (SWMM) using genetic parameter optimization. Urban Water Journal, v. 10, n. 6, p. 394-410, 2013. https://doi.org/10.1080/1573062X.2012.739631

LEVIA, D. F.; GERMER, S. A review of stemflow generation dynamics and stemflow-environment interactions in forests and shrublands. Reviews of Geophysics, v. 53, n. 3, p. 673-714, 2015. DOI: 10.1002/2015RG000479

LEVIA, D. F.; MICHALZIK, B.; NÄTHE, K.; BISCHOFF, S.; RICHTER, S.; LEGATES, D. R. Differential stemflow yield from European beech saplings: the role of individual canopy structure metrics. Hydrological Processes, v. 29, n. 1, p. 43-51, 2015. DOI: 10.1002/hyp.10124

LI, X.; NIU, J.; XIE, B. Study on hydrological functions of litter layers in North China. PLOS ONE, v. 8, n. 7, p. e70328, 2013. https://doi.org/10.1371/journal.pone.0070328

LI, X.; XIAO, Q.; NIU, J.; DYMOND, S.; VAN DOORN, N. S.; YU, X.; XIE B.; LV X.; ZHANG K.; LI, J. Process-based rainfall interception by small trees in Northern China: The effect of rainfall traits and crown structure characteristics. Agricultural and Forest Meteorology, v. 218-219, p. 65-73, 2016. https://doi.org/10.1016/j.agrformet.2015.11.017

LIVESLEY, S. J.; BAUDINETTE, B.; GLOVER, D. Rainfall interception and stemflow by eucalypt street trees - The impacts of canopy density and bark type. Urban Forestry e Urban Greening, v. 13, p. 192-197, 2014. https://doi.org/10.1016/j.ufug.2013.09.001

NANKO, K.; WATANABE, A.; HOTTA, N.; SUZUKI, M. Physical interpretation of the difference in drop size distributions of leaf drips among tree species. Agricultural and Forest Meteorology, v. 169, p. 74-84, 2013. DOI: 10.1016/j. agrformet.2012.09.018

SILVA, L. F.; LIMA, A. M. L. P.; SILVA FILHO, D. F.; COUTO, H. T. Z. Interceptação da chuva por duas espécies arbóreas em áreas verdes urbanas. CERNE, v. 16, p. 547-555, 2010. http://dx.doi.org/10.1590/S0104-77602010000400014

SOTO-SCHÖNHERR, S.; IROUMÉ, A. How much water do Chilean forests use? A review of interception losses in forest plot studies. Hydrological Processes, v. 30, n. 25, p. 4674-4686, 2016. DOI: 10.1002/hyp.10946

VAN DIJK, A. I.; GASH, J. H.; VAN GORSEL, E.; BLANKEN, P. D.; CESCATTI, A.; EMMEL, C.; GIELEN, B.; HARMAN, I. N.; KIELY, G.; MERBOLD, L.; MONTAGNANI, L.; MOORS, E.; SOTTOCORNOLA, M.; VARLAGIN, A.; WILLIAMS, C. A.; WOHLFAHRT, G. Rainfall interception and the coupled surface water and energy balance. Agricultural and Forest Meteorology, v. 214215, p. 402-415, 2015. https://doi.org/10.1016/j.agrformet.2015.09.006

XIAO, Q.; McPHERSON, E. G. Rainfall interception by Santa Monica's municipal urban forest. Urban Ecosystems, Davis, v. 6, p. 291-302, 2002.

XIAO, Q.; McPHERSON, E. G. Surface water storage capacity of twenty tree species in Davis, California. Journal of Environmental Quality, v. 45, n. 1, p. 188-198, 2016. https://doi.org/10.2134/jeq2015.02.0092

XIAO, Q.; McPHERSON, E. G.; USTIN, S. L; GRISMER, M. E. A new approach to modeling tree rainfall interception. Journal of Geophysical Research Atmospheres, v. 105, p. 29173-29188, 2000. DOI: 10.1029/2000JD900343

YANG, Y.; ENDRENY, T. A.; NOWAK, D. J. iTree-Hydro: snow hydrology update for the urban forest hydrology model. Journal of the American Water Resources Association, v. 47, p. 1211-1218, 2011. DOI: 10.1111/j.1752-1688.2011.00564.x 